\title{
THE USE OF ARTIFICIAL NEURAL NETWORKS IN CLINICAL MEDICINE
}

\author{
Miloš Jovanović \\ Dušan Milenković2, \\ Marija Perković, \\ Tatjana Milenković ${ }^{4}$, \\ Vuk Nikovićs \\ ${ }^{1}$ Singidunum University, \\ Belgrade, Serbia \\ ${ }^{2}$ Institute for Emergency Medical Care, \\ Niš, Serbia \\ ${ }^{3}$ Royal Holloway, University of London, \\ London, United Kingdom \\ ${ }^{4}$ Public Health Institute, \\ Niš, Serbia, \\ ${ }^{5}$ Institute for Emergency Medical Care, \\ Podgorica, Montenegro
}

\begin{abstract}
:
Digital Agenda in Serbia involves the introduction of an electronic system for monitoring of the main characteristics of patients, disease progression and treatment outcomes through EHR (Electronic Health Record). Internationally standardized data set contains more than 150 variables, with a tendency to introduce new frequently. In addition to the increased demand for treatment, there are also demands for optimizing the health care system. In order to predict the likelihood of diagnosis, course and outcome of treatment, classically multivariate regression linear logistic model is being used. In recent years, studies indicate that the use of Artificial Neural Networks (ANN) may provide improved results in terms of likelihood of final diagnosis and outcomes that include input variables which, by their nature, have a non-linear interdependence. We reviewed current ANN models, their advantages and disadvantages compared to common regression models and their applicability in clinical practice. Also, we analyzed and suggested models that could possibly optimize the process of diagnosis, predict the cost and duration of treatment and rationalize medical and other resources by reducing the cost/ benefit coefficient per patient.
\end{abstract}

Key words:

artificial neural networks, electronic health record, data mining.

\section{INTRODUCTION}

Digital Agenda in Serbia involves the introduction of an electronic system for monitoring of the main characteristics of patients, disease progression and treatment outcomes through EHR (Electronic Health Record) (Ministarstvo zdravlja, 2015). At the moment, it is estimated that the certified EHR covers approximately 50\% of health facilities, whereas the centralization of systems at the national level is currently in the implementation phase. In 2015, only in primary care, annual plan of preventive examination and for treatment of insured persons of all ages stood at over 45 million (RFZO, 2014). Such huge amount of data could be utilized for prediction of early accurate diagnosis, future adverse clinical events and treatment plans.

\section{Healthcare System Optimization}

In addition to the increased demand for treatment, there are also demands for optimizing the health care system. Decision to send patient for additional examinations or aggressive procedures is often based on 
clinical protocols adopted by consensus, mostly tailored by cost/benefit criteria (Wang, 2003). However, having in mind that most of them are linear logistic-based, it is unclear whether these protocols get the maximum predictability of aggregated data in real world settings.

\section{ELECTRONIC HEALTH RECORD (EHR)}

Internationally standardized data set for the Electronic Health Record (EHR) contains more than 150 variables, with a tendency to introduce new frequently. It is based on ISO/HL7 Standards and provides various baseline and input variables. It contains administrative, socio-medical, general health data (main set - previous diseases, special problems, immunizations, risk factors, drugs, contact with healthcare system anywhere), organ recipient data, primary cause of death and additional metadata (Ministarstvo zdravlja RS, 2009).

Basically, EHR contains "raw" data aggregated initially and over a certain period, as patient's contact with healthcare providers sums up. Such huge data sets might represent an excellent foundation for further analysis and possibly clinical algorithms development.

\section{MULTIVARIATE REGRESSION LINEAR LOGISTIC MODEL}

To predict the likelihood of diagnosis, course and outcome of treatment, classically multivariate regression linear logistic model is being used (Sobb, 2008).

Since there is a linear relationship between variables, these regression models tend to yield mixed results in modeling some complex connections and relations, as seen in very diverse and multifactorial biologic systems (DiRusso, 2002).

On he other hand, when it comes to discovery of underlying relationships between variables, linear regression seems to be method of choice for modeling. On the weak side, such models demonstrate lower potential at predicting likelihood of final events during such complex interactions (Lin, 2010).

\section{ARTIFICIAL NEURAL NETWORKS (ANN)}

In recent years, studies show that the use of Artificial Neural Networks (ANN) may provide some quality results considering sensitivity and specificity of final diagnosis and outcomes that include input variables which, by their nature, have a non-linear interdependence (Casagranda 2016, Freeman 2000, DiRusso 2002). Performance of ANN modeling is speculated to be superior than standard statistical methods in many scientific fields. Particularly, in medical research, there are growing number of reports where ANN modeling provide better results compared to Cox regression, discriminant analysis, and logistic regression (Eftekhar, 2005).

Applications of ANNs in medical and healthcare fields cover many clinical and laboratory procedures. ANNs is found to be convenient tool for predicting outcomes and mortality in trauma patients (Eftekhar, 2005; Abbod, 2011), in patients after invasive cardiac procedures (Freeman, 2000), for detecting abdominal diseases (Liew, 2007), for determining risk of serious conditions in emergency departments (Casagranda, 2016), for medical image studies (Jiang 2010), for evaluating cancer screening programs (Álvarez Menéndez, 2010), for correct interpretation of laboratory and intensive care results (Guo, 2010), and many more.

Recent papers suggested the use of ANNs in evaluating implementation model of remote health observing based on suitable questionnaire (Huang, 2010) and implementing web-based health monitoring systems for spread use by patients in their home environment (Youm, 2011).

Similar to weather forecasting methods, ANNs could find their potential use in forecasting of overcrowding in emergency departments (Jones 2009), operating rooms or be utilized as a tool for proper positioning of ambulance vehicles in large density population areas (Nguyen, 2015). The strongest advantage of ANNs over logistic regression in these areas of research may be due to ANN's capability to explore some relations between variables and the outcomes that are uncertain or poorly understood at the moment.

\section{Analyzing big data sets and data selection}

As number of data collected in healthcare system tend to rise over time, physician's ability to recognize important findings and certain patterns in overall patient's clinical condition tends to drop. Such amount of data may be sometimes hard to synthesize in a form of meaningful outcome, especially with mixed variable sets (Ghavami, 2012).

There is ongoing debate whether ANN could or should be used in each clinical settings. Growing number of published papers and elevated interest in ANN as a tool for finding relations among apparently completely 
unrelated input variables for predicting clinical outcomes raised some doubt considering validity of results (Freeman, 2000). To avoid such speculations, it is may be beneficial to build ANN models on initial subset of univariate predictors based on mathematical algorithms and established clinical knowledge.

Selecting proper variables is generally based on empirical data and basic statistical methods which identifies variables with strongest predictive powers (Ghavami, 2012).

\section{Basic ANN structure}

Standard design of ANN is based on several mathematically-driven nodes structured in several respective layers (Fig. 1). Separate node is linked to all nodes in preceding layer. In general, ANN structure is based on three layers: input layer (denotes input variable), "hidden" layer (denotes computation processing) and output layer (denotes outcome for the specified data record). Each ANN model consists of training and validation datasets.

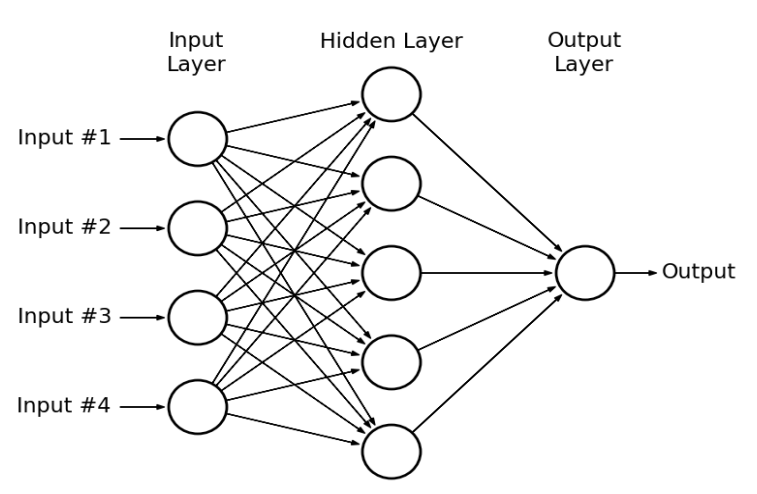

Fig. 1. Common ANN structure

Training sets are built with sequence of input variables with known outcomes. These data are commonly purely empirical (Amato, 2013). Variables at each input nodes are given randomly calculated numeric attribute values ("weights"). In the next step, the sum of weights of all input data is computed and introduced to every single node in the hidden layer. Finally, using transfer function, the weighted sum is utilized to calculate the power of single node's output by assigning it a scaled numerical value (Yamamura, 2003). Long story short, properly designed network has ability to "learn" from a sequence of "examples" contained in the training database.
After the training process is done, the neural network can be tested and verified on new external data provided with only input values. Once verified by clinicians or researchers, the new data can be added to existing database for further training, but only if they belong to the same observed cohort indicated by appropriate parameter value (Amato, 2013).

\section{ANN limitations}

The configuring of an ANN is mostly an empirical process, so there are no specific consensus recommendations for designing of an optimal ANN. Large amount of input variables are initially chosen at random and put to test. The final models are usually selected on the basis of desired cut-off probability values for expected events. ANN modeling is currently discussed as a form of "black box", for the reason that the correlations between the selected cluster of variables that make the correct output are not properly understood (Benitez, 1997).

\section{EHR databases as a platform for modeling ANN}

EHR was initially designed as a tool for collecting relevant patient's data over his lifetime with objective to improve attending physician's decisions regarding previous, current and future treatment plans for specific health conditions, and to follow up overall medical costs. The extent of EHR use has risen in many countries over the past decade, so naturally, the number of data stored surpassed human's ability to review and extract applicable information out of so much data considering treatment choice. In recent years, noticeable interest has raised addressing possible use of ANN and other methods in data mining of EHR, specifically in genetics and family health history (Ross, 2014; Hoyt, 2016).

At the moment, clinical trials symbolize reference methods in making policies for developing and introducing new drugs, therapeutic procedures, clinical algorithms and more, enrolling on average few hundred or thousand subjects. Although the bias is excluded by subject randomization to receive or not certain treatment, conclusions made at the end of investigations are result of basically "noise reduction" by carefully selecting cohort with desired baseline parameters. One of the questions unanswered, even with the large registries of events, is what other variables, omitted from the investigation, influence real-life performance of derived drug and therapeutic guidelines found in observational studies 
(Zhang, 2014; Balas, 2015). EHR data mining found some promising results that could improve clinical trials' correct preparation or proper subject selection (Ross, 2014).

EHR databases contain many input variables from various patients, but not all of them are at the same time given certain value, i.e. they remain empty until operator/physician interacts and inputs the data (laboratory findings, for example). One of the problem that should be addressed is that "missing values" could interfere with expected ANN results, so the correct database pre-processing should be done. This could be more complicated when introducing new input variables, as "over-fitting" may occur because of ANN's tendency to pair inputoutput data too precisely. This can be resolved by crossvalidation of input variables in a parallel clone database.

Another issue could be the determining proper number of iterations ("epochs") that are required for ANN to reach it's optimized state. As this process can be timeconsuming and varies with different ANNs used and verified (based on number of input variables, number of hidden layers and hidden nodes that each of them contains), and is set by user threshold that is reached for the mean square error change, cloud-based computational engines utilization for such fair amount of data processing seems to be the pathway to look after in these scenarios.

\section{ANNs for remote health monitoring}

As the portable diagnostic equipment evolve (such as portable ECGs and holter devices, blood glucose analyzers), there is an elevated interest in utilizing recorded data for forecasting adverse events in certain high-risk and chronic patients. ANNs have already shown some improved results compared to mainstream logistic regression algorithms (Radhimeenakshi 2015). Sensors readings are transmitted over cellular lines or $\mathrm{Wi}-\mathrm{Fi}$ networks, and together with patient's baseline known input characteristics and ANN trained dataset from large registries, aggregated data are processed for likelihood of heart failure, preterm birth or poor diabetes control. ANNs may be used to trigger alarms before critical events, or to imply clinician's decision to correct underlying cause of disturbance.

Building a suitable database may be achieved also by entering required data on a daily basis by patients themselves (Suh, 2012). Such data may be included as a separate variable list in an addendum to EHR. Specific online questionnaires are fed with current patient's data, such as daily symptoms (chest pain, headache, mood changes, etc.), calories intake, sleeping periods, heart rate, blood pressure and many more, and the suitable ANN model reacts on threshold parameters. On the other hand, many of these ANN applications are prone to errors if missing data are not processed properly or data do not undergo supervised learning, so it's maintenance complexity often comes as a deal-breaker for long term reliable conclusions and deployment.

\section{ANNs for Clinical Decision Support Systems (CDSS)}

CDSS represent heterogeneous group of healthcare software and hardware largely built on rule-based statistical theory and Arden syntax that combine existing clinical knowledge (in a form of widely accepted guidelines or consensus papers) with physician's input to suggest further laboratory or procedure ordering, as seen in many EHR-based systems. One of the CDSS drawbacks is that suggested sets of laboratory tests or procedures are group-oriented, rather than patient-oriented sets (Castaneda, 2015).

Having also in mind that the most of the current CDSS are prone to errors and malfunctions (Wright, 2016), ANNs may find their role in future CDSS designs aimed for patient-oriented or customized clinical decisions on patient-by-patient basis (Kyrgiou, 2015). Furthermore, CDSS hybrid data-driven and rule-based systems (e.g. multilayer neural network and C4.5. decision tree algorithm) could have the potential to return better results in the future (Bal, 2014).

\section{Future directions for ANN implementation}

Aside of ANN's power to predict outcomes based on initial dataset, diversity and complexity combined with huge number of possible variables makes ANN sometimes difficult to implement in real practice. On the other hand, one could always argue the strength of actual variables, as opposed to missed one's in the datasets. One step further would be, for example, the designing of ANNs to suggest which optimal number and type of input variables is needed to obtain fairly precise diagnosis or outcome of particular patient's condition based on a few clinical symptoms and patient's baseline characteristics.

\section{COST/BENEFIT ANALYSIS}

Introducing EHR already showed decline in overall healthcare expenditure (Wnag, 2003). Clinician's decision to send patient for further analysis or interventional pro- 
cedures generally raises funds needed for managing patient's condition. There are several studies that addressed this problem, since additional tests and procedures do not always result in definitive diagnosis or termination of illness. There are several studies that suggest the use of ANN models in reducing overall costs for proper treatment of clinical conditions (Walczak, 2000; Liew, 2007, Abbod, 2011; Teferra, 2014).

It may be reasonable to reveal real power of ANNs in minimizing healthcare costs by widespread implementation in screening programs, as seen in primary health care. Major savings are known to be results of early detection and proper management of diseases. Although there are many well established screening programs aimed for diseases with greater incidence or prevalence among selected population (cancer, cardiovascular and metabolic diseases), main advantage of ANNs lies in ability to detect some unusual patterns in disease origin and progression, as well as to produce fewer number falsepositive predictions.

Costs reduction and benefit optimization also may be found in proper ANN-derived strategic planning of healthcare personnel engagement during peak days or hours, proper geo-data positioning of ambulance vehicles, threshold settings for decisions to enter operating room, to have CT or MRI done, for entering program of in vitro fertilization, and many more.

Current strategies are not consistent across countries, as healthcare budgets are not equal. It may be reasonable, for developing countries, to establish their own models based on EHR data sets and algorithms matching healthcare funds per patient and desired disease detection and control. Also, one should bare in mind that socio-epidemiological settings play important role in determining quality of life and subsequent emerging of health problems. Chronic stress, quantifiable or not by ANN, remains inevitable trigger for many diseases.

\section{CONCLUSION}

With the development of microprocessor and cloud technology, mega-data sets can now be analyzed within a reasonable time by finding the model ANN that can optimize the process of diagnosis, predict the cost and duration of treatment and rationalize medical and other resources by reducing the cost/benefit coefficient per patient.
Logistic regression models seem to complement for major drawback of ANNs, in terms of finding relations between individual factors that determine proper data selection.

Further EHR dataset analysis using ANN could potentially improve heal thcare system performance by "learning" global health tendencies among observed population.

\section{REFERENCES}

Abbod, M., Cheng, K., Cui, X., Huang, S., Han, Y., \& Shieh, J. (2011). Ensembled neural networks for brain death prediction for patients with severe head injury. Biomedical Signal Processing And Control, 6(4), 414-421. DOI:10.1016/j.bspc.2011.01.002

Álvarez Menéndez, L., de Cos Juez, F., Sánchez Lasheras, F., \& Álvarez Riesgo, J. (2010). Artificial neural networks applied to cancer detection in a breast screening programme. Mathematical And Computer Modelling, 52(7-8), 983-991. DOI:10.1016/j. mcm.2010.03.019

Amato, F., López, A., Peña-Méndez, E., Vaňhara, P., Hampl, A., \& Havel, J. (2013). Artificial neural networks in medical diagnosis. Journal Of Applied Biomedicine, 11(2), 47-58. DOI:10.2478/v10136012-0031-x

Bal, M., Amasyali, M., Sever, H., Kose, G., \& Demirhan, A. (2014). Performance Evaluation of the Machine Learning Algorithms Used in Inference Mechanism of a Medical Decision Support System. The Scientific World Journal, 2014, 1-15. DOI:10.1155/2014/137896

Balas EA, Vernon M, Magrabi F, Gordon LT, Sexton J. (2015). Big Data Clinical Research: Validity, Ethics, and Regulation. Stud Health Technol Inform, 216:448-52. DOI:10.3233/978-1-61499-564-7-448

Benitez, J., Castro, J., \& Requena, I. (1997). Are artificial neural networks black boxes?. IEEE Trans. Neural Netw., 8(5), 1156-1164. DOI:10.1109/72.623216

Casagranda, I., Costantino, G., Falavigna, G., Furlan, R., \& Ippoliti, R. (2016). Artificial Neural Networks and risk stratification models in Emergency Departments: The policy maker's perspective. Health Policy, 120(1), 111-119. DOI:10.1016/j.healthpol.2015.12.003

Castaneda, C., Nalley, K., Mannion, C., Bhattacharyya, P., Blake, P., \& Pecora, A. et al. (2015). Clinical decision support systems for improving diagnostic accuracy and achieving precision medicine. J Clin Bioinform, 5(1). DOI:10.1186/s13336-015-0019-3 
DiRusso, S., Chahine, A., Sullivan, T., Risucci, D., Nealon, P., \& Cuff, S. et al. (2002). Development of a model for prediction of survival in pediatric trauma patients: Comparison of artificial neural networks and logistic regression. Journal Of Pediatric Surgery, 37(7), 1098-1104. DOI:10.1053/jpsu.2002.33885

Eftekhar, B., Mohammad, K., Ardebili, H., Ghodsi, M., \& Ketabchi, E. (2005). Comparison of artificial neural network and logistic regression models for prediction of mortality in head trauma based on initial clinical data. BMC Med Inform Decis Mak, 5(1), 3. DOI:10.1186/1472-6947-5-3

Freeman, R., Eagle, K., Bates, E., Werns, S., Kline-Rogers, E., Karavite, D., \& Moscucci, M. (2000). Comparison of artificial neural networks with logistic regression in prediction of in-hospital death after percutaneous transluminal coronary angioplasty. American Heart Journal, 140(3), 511-520. DOI:10.1067/mhj.2000.109223

Ghavami, P. (2012). An Investigation of Applications of Artificial Neural Networks in Medical Prognostics (Ph.D.). University of Washington. Retrievd March 16, 2016. from https://digital.lib.washington.edu/researchworks/bitstream/handle/1773/21917/Ghavami_washington_0250E_10779.pdf?sequence $=1$

Guo, L., Rivero, D., Dorado, J., Rabuñal, J., \& Pazos, A. (2010). Automatic epileptic seizure detection in EEGs based on line length feature and artificial neural networks. Journal Of Neuroscience Methods, 191(1), 101-109. DOI:10.1016/j.jneumeth.2010.05.020

Hoyt, R., Linnville, S., Thaler, S., \& Moore, J. (2016). Digital Family History Data Mining with Neural Networks: A Pilot Study. Perspect Health Inf Manag, 13(Winter), 1c. Retrieved from http://www.ncbi.nlm.nih. gov/pmc/articles/PMC4739442/pdf/phim00130001c.pdf

Huang, J. (2010). Remote health monitoring adoption model based on artificial neural networks. Expert Systems With Applications, 37(1), 307-314. DOI:10.1016/j.eswa.2009.05.063

Jiang J, Trundle P, Ren J. (2010) Medical image analysis with artificial neural networks. Comput Med Imaging Graph, 34(8):617-31. DOI: 10.1016/j.compmedimag.2010.07.003.

Jones, S., Evans, R., Allen, T., Thomas, A., Haug, P., Welch, S., \& Snow, G. (2009). A multivariate time series approach to modeling and forecasting demand in the emergency department. Journal Of Biomedical Informatics, 42(1), 123-139. DOI:10.1016/j.jbi.2008.05.003

Kyrgiou, M., Pouliakis, A., Panayiotides, J., Margari, N., Bountris, P., \& Valasoulis, G. et al. (2016). Personalised management of women with cervical abnormalities using a clinical decision support scoring system. Gynecologic Oncology, 141(1), 29-35.DOI:10.1016/j. ygyno.2015.12.032
Liew, P., Lee, Y., Lin, Y., Lee, T., Lee, W., Wang, W., \& Chien, C. (2007). Comparison of artificial neural networks with logistic regression in prediction of gallbladder disease among obese patients. Digestive And Liver Disease, 39(4), 356-362. DOI:10.1016/j. dld.2007.01.003

Lin, CC., Ou, YK., Chen, SH., Liu, YC., Lin, J. (2010). Comparison of artificial neural network and logistic regression models for predicting mortality in elderly patients with hip fracture. Injury 41(8), 869-873.

May, R., Dandy, G., \& Maier, H. (2011). Review of Input Variable Selection Methods for Artificial Neural Networks. Artificial Neural Networks - Methodological Advances And Biomedical Applications. DOI:10.5772/16004

Ministarstvo zdravlja RS (2009). Pravilnik o bližoj sadržini tehnoloških i funkcionalnih zahteva za uspostavljanje integrisanog zdravstvenog informacionog sistema. Retreived March 15, 2016. http://www.rfzo.rs/ download/pravilnici/mz/Pravilnik_integrisanizdrsistem.pdf. Article in Serbian.

Ministarstvo zdravlja RS (2015). EU-IHIS Integrisni informacioni sistem. Pregled projekta. Retreived March 15, 2016. from http://www.eu-ihis.rs/docs/Docs/ TechDocs/EU-IHIS_Pregled\%20projekta_Konacan_SR.pdf. Article in Serbian.

Nguyen, N. (2015). Quantitative Analysis of Ambulance Location-allocation and Ambulance State Prediction. Norrköping: Department of Science and Technology, Linköping University. Retrieved March 16, 2016. from http://liu.diva-portal.org/smash/get/ diva2:781472/FULLTEXT01.pdf

Radhimeenakshi, S., \& Nasira, G. (2015). Remote Heart Risk Monitoring System based on Efficient Neural Network and Evolutionary Algorithm. Indian Journal Of Science And Technology, 8(14). DOI:10.17485/ ijst/2015/v8i14/72732

RFZO (2014). Plan zdravstvene zaštite iz obaveznog zdravstvenog osiguranja u Republici Srbiji za 2015. godinu. Retreived March 15, 2016. from http:// www.rfzo.rs/download/Plan\%20zdravstvene\%20 zastite\%20za\%202015.pdf. Article in Serbian.

Ross, M., Wei, W., \& Ohno-Machado, L. (2014). "Big Data" and the Electronic Health Record. IMIA Yearbook, 9(1), 97-104. DOI:10.15265/iy-2014-0003

Sobh, M., Cleophas, T., Hadj-Chaib, A., \& Zwinderman, A. (2008). Clinical Trials: Odds Ratios and Multiple Regression Models-Why and How to Assess Them. American Journal Of Therapeutics, 15(1), 44-52. DOI:10.1097/mjt.0b013e3180ed80bf

Suh, M. (2012). Dynamic adaptive remote health monitoring for patients with chronic disease (Ph.D.). University of California, Los Angeles. 
Teferra, R., Grant, B., Mindel, J., Siddiqi, T., Iftikhar, I., \& Ajaz, F. et al. (2014). Cost Minimization Using an Artificial Neural Network Sleep Apnea Prediction Tool for Sleep Studies. Annals ATS, 11(7), 10641074. DOI:10.1513/annalsats.201404-161oc

Walczak, S., \& Scharf, J. (2000). Reducing surgical patient costs through use of an artificial neural network to predict transfusion requirements. Decision Support Systems, 30(2), 125-138. DOI:10.1016/s01679236(00)00093-2

Wang, S., Middleton, B., Prosser, L., Bardon, C., Spurr, C., \& Carchidi, P. et al. (2003). A cost-benefit analysis of electronic medical records in primary care. The American Journal Of Medicine, 114(5), 397-403. DOI:10.1016/s0002-9343(03)00057-3

Wright, A., Hickman, T., McEvoy, D., Aaron, S., Ai, A., \& Andersen, J. et al. (2016). Analysis of clinical decision support system malfunctions: a case series and survey. Journal Of The American Medical Informatics Association, ocw005. DOI:10.1093/jamia/ocw005
Yamamura, S. (2003). Clinical application of artificial neural network (ANN) modeling to predict pharmacokinetic parameters of severely ill patients. Advanced Drug Delivery Reviews, 55(9), 1233-1251. DOI:10.1016/s0169-409x(03)00121-2

Youm, S., Lee, G., Park, S., \& Zhu, W. (2011). Development of remote healthcare system for measuring and promoting healthy lifestyle. Expert Systems With Applications, 38(3), 2828-2834. DOI:10.1016/j. eswa.2010.08.075

Zhang Z. (2014). Big data and clinical research: perspective from a clinician. J Thorac Dis. 6(12): 1659-1664. DOI:10.3978/j.issn.2072-1439.2014.12.12 